\title{
Variable Momentum Controlled by Equalizer Energy based CMA Blind Equalization \\ Jinyu SUN ${ }^{\mathrm{a}}$, Ying XIAO ${ }^{\mathrm{b}}$
}

College of Information and Communication Engineering, Dalian Nationality University, Dalian 116600, China

a2440452893@qq.com, bxiaoying@126.com

\begin{abstract}
Keywords: blind equalization; CMA; momentum; underwater acoustic channel
\end{abstract}
\begin{abstract}
To further improve the performance of CMA blind equalization, an improved algorithm with variable momentum controlled by equalizer weights energy was proposed. The momentum gradient algorithm can improve the convergence rate effectively. However the additional gradient noise would produce by momentum, which results in high steady state residual error after convergence. Hereby, a variable momentum algorithm is designed according to the equalizer weights energy, which the momentum factor adjusted needs no empirical parameters during the iterative process. Therefore, the proposed algorithm is more universal than the other adaptive momentum algorithms. The variable momentum controlled by equalizer weights energy not only can take advantage of the momentum gradient algorithm to improve the convergence rate, but also can avoid the additional gradient noise produce by momentum for CMA blind equalization, and the effectiveness is shown by computer simulation under underwater acoustic channel condition.
\end{abstract}

\section{Introduction}

Blind equalization can compensate and track the communication channel without the training signal, which can remove the inter-symbol interference in the received signal to improve the quality of the communication and save the bandwidth [1], at the same time, it can avoid the unlock of the equalizer effectively. Recently, lots of research achievements of the theory and algorithm of blind equalization have obtained by the relevant experts and scholars. In all kinds of blind equalization algorithms, CMA blind equalization structure is simple and the performance is stable. However, the convergence rate of CMA is slow and the steady state residual error is big. Based on CMA blind equalization, lots of improved algorithms were proposed, which include the momentum CMA blind equalization algorithm. The momentum gradient algorithm can accelerate the convergence rate, and it can avoid falling into the shallow local minimum of the cost function to improve the global convergence performance when the cost function is non-convex [3]. However, the momentum produces additional gradient noise after the algorithm convergence, which leads to the increase of the residual error. To overcome the effect of momentum term on the residual error, the adaptive momentum algorithm is considered as a compromise algorithm in the convergence rate and convergence accuracy. The basic idea of the adaptive momentum algorithm is that the big momentum factor is used to obtain fast convergence rate at the initial stage, and the momentum factor is gradually reduced as the algorithm iterative process to obtain small steady state residual error. The adaptive momentum algorithms proposed by the relevant literature need to design the empirical parameters, for different communication channels of different SNR conditions, the algorithm is poor, and the design of empirical parameters is lack of theoretical basis, such as statistical momentum factor algorithm and adaptive momentum factor controlled by the function of instantaneous error nonlinear transformation. Hereby, an improved algorithm with variable momentum controlled by equalizer weights energy was proposed, variable momentum algorithm is designed according to the equalizer weights energy, which the momentum factor adjusted needs no experience parameters during the iterative process. Therefore, the proposed algorithm is more universal than the other adaptive momentum algorithms. The effectiveness of the proposed algorithm is shown by computer simulation under underwater acoustic channel condition. 


\section{The Basic Principle of CMA}

The basic principle of CMA is shown in Fig.1 [4]. Where $\boldsymbol{x}(n)$ is the input signal, $\boldsymbol{h}(n)$ is the unknown channel impulse response, $\boldsymbol{n}(n)$ is the Gaussian white noise with zero mean, $\boldsymbol{y}(n)$ is the received signal and $\boldsymbol{w}(n)$ is the blind equalizer weights and $\tilde{x}(n)$ is the output signal of the blind equalizer.

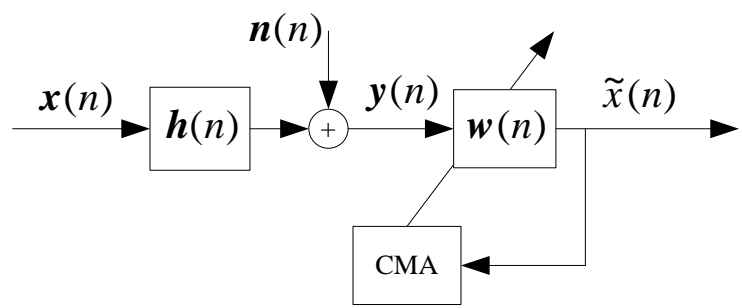

Fig. 1 The basic principle of CMA

According to the communication signal transmission process can be known

$$
\begin{aligned}
& \boldsymbol{y}(n)=\boldsymbol{h}(n) \boldsymbol{x}(n)+\boldsymbol{n}(n) \\
& \tilde{x}(n)=\boldsymbol{w}^{H}(n) \boldsymbol{y}(n)
\end{aligned}
$$

CMA blind equalization uses the high order statistics of the transmission signal and its cost function can be given by [5]

$$
J(n)=\frac{1}{2}\left[R-|\tilde{x}(n)|^{2}\right]^{2}
$$

Where $R$ is the constant modulus of the transmission signal which is given by

$$
R=\frac{E\left[|\tilde{x}(n)|^{4}\right]}{E\left[|\tilde{x}(n)|^{2}\right]}
$$

According to the stochastic gradient descent algorithm, the updating formula of CMA blind equalization can be given by

$$
\boldsymbol{w}(n+1)=\boldsymbol{w}(n)+\mu e(n) \boldsymbol{y}^{*}(n) \tilde{x}(n)
$$

Where $\mu$ is the study step and $e(n)$ is the instantaneous gradient error which can be given by

$$
e(n)=R-|\tilde{x}(n)|^{2}
$$

\section{Momentum CMA Blind Equalization}

The convergence rate of CMA blind equalization is slow, which would waste lots of symbol information to capture the communication channel characteristics, as a result, it causes the error rate in the receiving signal is too high especially in high speed communication or time varying channel conditions. Furthermore, the tracking ability of the channel of CMA blind equalization is reduced sharply. Momentum gradient descent algorithm can significantly improve the convergence rate, while it can avoid the shallow local minimum, so as to improve the global convergence performance of the algorithm for the non-convex cost function. The blind equalizer weights updating formula of blind equalizer based on momentum gradient can be given by [6]

$$
\boldsymbol{w}(n+1)=\boldsymbol{w}(n)+\mu e(n) \boldsymbol{y}^{*}(n) \tilde{x}(n)+\alpha[\boldsymbol{w}(n)-\boldsymbol{w}(n-1)]
$$

Where $0<\alpha<1$ is the momentum factor and $\alpha[w(n)-w(n-1)]$ is the momentum term. Let $\Delta \boldsymbol{w}(n)=\boldsymbol{w}(n+1)-\boldsymbol{w}(n)$, then the recurrence relation can be given by Eq.8 according to Eq.7.

$$
\Delta \boldsymbol{w}(n)=\mu e(n) \boldsymbol{y}^{*}(n) \tilde{x}(n)+\alpha \Delta \boldsymbol{w}(n-1)
$$

Expanding Eq.8 can obtain the result as follow

$$
\Delta \boldsymbol{w}(n)=\sum_{i=1}^{n-1} \alpha^{n-i} \nabla J(i)+\alpha^{n}(\boldsymbol{w}(1)-\boldsymbol{w}(0))
$$

Where $\nabla J(n)$ is the instantaneous gradient of CMA. Eq.9 shows that the momentum factor must 
meet the condition as $|\alpha|<1$ to ensure the convergence of the algorithm. For the momentum cannot rapid the convergence rate when $\alpha<0$, and then the range of momentum factor is $0<\alpha<1$. If the CMA blind equalization meets the ideal convergence condition, it needs $\Delta \boldsymbol{w}(n)=\boldsymbol{0}$. According to Eq.7, when CMA blind equitation reaches to ideal convergence rate, the momentum term would produce additional gradient noise, which increases the steady state residual error.

Adaptive momentum gradient algorithm uses the bigger momentum factor in the initial stage of the blind equalizer updating process, which can rapid the convergence rate. With the iterative of the algorithm, the momentum factor reduces gradually to obtain smaller steady state residual error. Thus the adaptive momentum gradient algorithm can obtain a compromise result between convergence rate and convergence accuracy. Some adaptive momentum algorithms can be given by follows [7-9].

$$
\begin{aligned}
& \alpha(n)=\beta e(n) / \sum_{j=1}^{n} e(j) \\
& \alpha(n)=\left\{\begin{array}{cc}
\frac{2}{3} & E(n) \geq 2 \\
E(n) / 3 & E(n)<2
\end{array}\right. \\
& \alpha(n)=\gamma\left[1-e^{p-E(n)}\right] \\
& \alpha(n)=\gamma\left[1-e^{p-|e(n)|^{2}}\right]
\end{aligned}
$$

Where $\beta, \gamma$ and $p$ are empirical parameters and the reasonable design of empirical parameters has a significant effect on the performance of the algorithm. Although the adaptive momentum algorithm given by Eq.11 without empirical parameters, the factor is $2 / 3$ or $2 / 3 E(n)$ without the theoretical basis. Furthermore, it cannot obtain ideal convergence performance for different SNR or different type modulate signal. Where the estimation method of $E(n)$ is given by

$$
E(n+1)=\lambda E(n)+(1-\lambda)|\tilde{x}(n)-\hat{x}(n)|^{2}
$$

Where $\lambda$ is the forgetting factor and $\hat{x}(n)$ is the decision value of $\tilde{x}(n)$.

The nature of CMA blind equalization is stochastic gradient descent algorithm [10], which achieves the ideal equalization by minimizing the cost function. The instantaneous gradient variation rate is zero and the variation rate of the energy of the equalizer weight is zero when the algorithm meets ideal equalization condition without taking into account the effect of the noise in the channel. The variation rate of the equalizer weights is defined as follow.

$$
\Delta \boldsymbol{w}(n)=\boldsymbol{w}(n)-\boldsymbol{w}(n-1)
$$

After the algorithm obtains convergence, it can get the following conclusion.

$$
\lim _{n \rightarrow \infty}(\Delta \boldsymbol{w}(n)-\Delta \boldsymbol{w}(n-1))=\mathbf{0}
$$

Then the energy of the equalizer weights is defined as follow.

$$
E_{w}(n)=\sum_{j=1}^{L}\left|w_{j}(n)\right|
$$

Where $L$ is the length of the equalizer. According to Eq.16 we can get

$$
\lim _{n \rightarrow \infty} E_{w}(n)=\lim _{n \rightarrow \infty} E_{w}(n-1)
$$

According to Eq.16 and Eq.18 we can get

$$
\lim _{n \rightarrow \infty} \Delta E_{w}(n)=\lim _{n \rightarrow \infty}\left(E_{w}(n)-E_{w}(n-1)\right)=0
$$

Then the variable momentum CMA blind equalization algorithm based on the equalizer weights energy can be obtained and the momentum factor can be adjusted by

$$
\alpha(n+1)=\alpha(n)+\mu_{m}\left[\frac{\left|\Delta E_{w}(n)\right|-\left|\Delta E_{w}(n-1)\right|}{\max \left(\left|\Delta E_{w}(n)\right|,\left|\Delta E_{w}(n-1)\right|\right)}\right]
$$


Where $\mu_{m}$ is the study step of the momentum factor, $\max (a, b)$ represents the maximum value of $a$ and $b$. In the initial stage of the algorithm, $\Delta E_{w}(n)$ varies during the iterative process and $\Delta E_{w}(n)$ would larger than $\Delta E_{w}(n-1)$ in a statistical sense. And then the momentum factor adjusted according to Eq.20 can get a gradually reduced process. Considering the increase of the iterative process, the momentum factor may meet $\alpha(n)<0$. Therefore, the variation of momentum factor is constrained by the following formula.

$$
\alpha(n+1)=\alpha(n) \quad \text { if } \quad \alpha(n+1)<0
$$

The variable momentum algorithm according to Eq.20 and Eq.21 called energy control variable momentum CMA (ECVMCMA). There are no empirical parameters in ECVMCMA, which can let the proposed algorithm is more universal than the other adaptive momentum algorithms.

\section{Computer Simulation and Analysis}

In the simulation, the send signal is modulated by QPSK. The channel is adopted the mix-phase underwater acoustic channel. The channel equivalent baseband impulse response is $h=[0.3132,0.1040,0.8908,0.3143]$ [11]. The channel noise is Gauss white noise with zero mean and the SNR=20dB. The length of the blind equalizer is 16 and the center weight is initialized to 1 and the other weights are initialized to 0 . The study step of the equalizer $\mu=0.001$ and the study step of the momentum factor $\mu_{m}=0.002$. The initial value of the momentum factor $\alpha(0)=0.75$. The performance comparison is evaluated according to residual inter symbol interference (ISI) which is defined as follow [12].

$$
\operatorname{ISI}(n)=\frac{\sum_{i}\left|C_{i}(n)\right|^{2}-\left|C_{i}(n)\right|_{\max }^{2}}{\left|C_{i}(n)\right|_{\max }^{2}}
$$

Where $\boldsymbol{C}(n)$ is the combined impulse response vector. The residual ISI convergence result after 500 times Monte Carlo simulations is shown in Fig.2 and the variation curve of the momentum factor is shown in Fig.3.

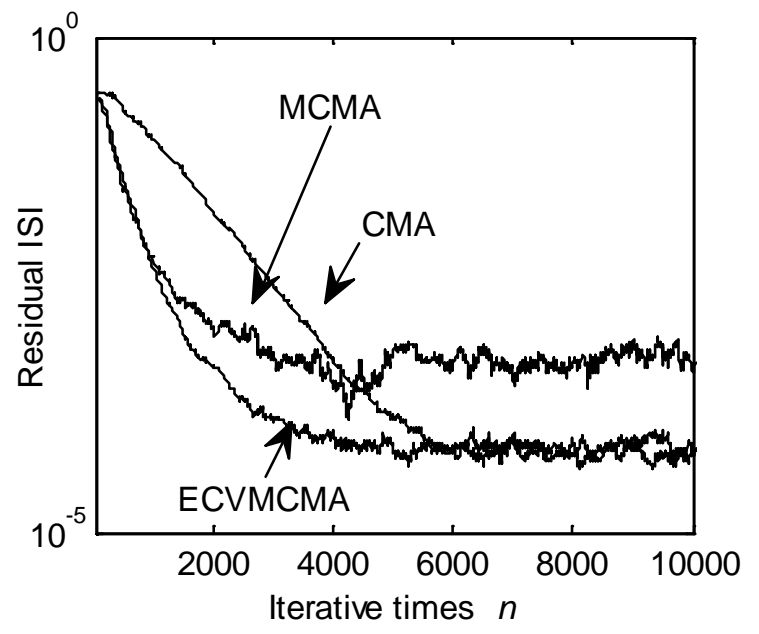

Fig. 2 The residual ISI convergence curve

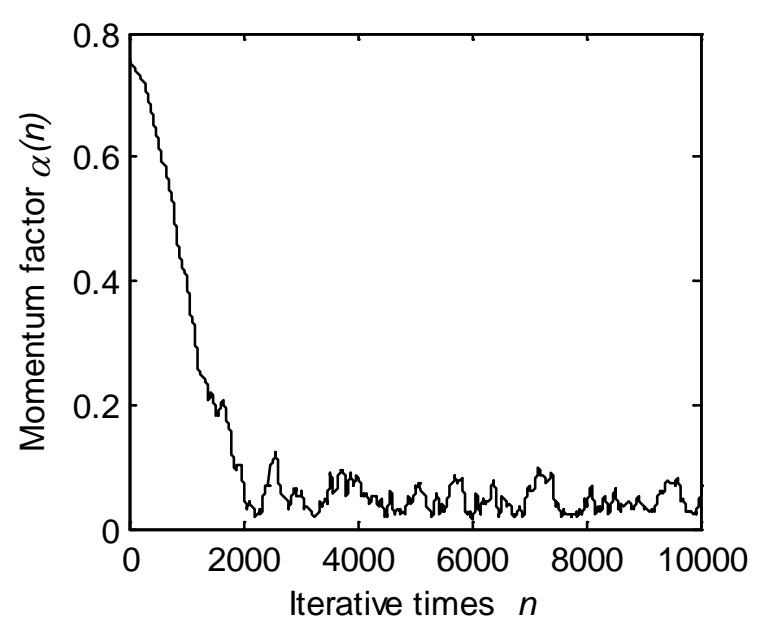

Fig.3 The variation curve of momentum factor

Fig. 2 shows that the proposed ECVMCMA blind equalization algorithm has faster convergence rate than the traditional CMA and has the consistent steady stable residual error with the traditional CMA after convergence. The momentum CMA (MCMA) with the constant momentum factor has the fastest convergence rate, but the steady stable residual error is bigger due to the additional gradient noise produced by momentum. Fig. 3 shows that the momentum factor reduces gradually with the iterative process and convenience to a small value near to 0 after 2000 times iterative, thus it can avoid the additional gradient noise produced by momentum. 


\section{Conclusion}

The momentum CMA blind equalization can improve the convergence rate of the algorithm effectively. However, the additional gradient noise produced by momentum increases the steady stable residual error after convergence. Adaptive momentum CMA blind equalization algorithms often need empirical parameters, which results in the degeneration of the universal. This work proposed a variable momentum CMA blind equalization algorithm, which the momentum factor updates according to the variation rate of the energy of the blind equalizer weights and there is no empirical parameters need to set, therefore, the proposed algorithm improves the universal for adaptive momentum CMA. The simulation results show that the proposed algorithm has the approaching convergence rate with MCMA and has the approaching convergence accuracy with the traditional CMA.

\section{Acknowledgement}

This work is supported in part by The National Natural Science Foundation of China (61201418), Fundamental Research Funds for the Central Universities (DC201502060302) and Liaoning Province High School Talent Support Program (LJQ2013126).

\section{References}

[1] Mendes Filho J., Miranda M.D., Silva M.T.M. Decision-directed feedback equalisation for QAM signals [J]. Electronics Letters, 2012, 48(1): 27-28.

[2] Y.Sato. A method of self-recovering equalization for multi-level amplitude modulation [J]. IEEE Transactions on Communication, 1975, 23(6): 679-682.

[3] XIAO Ying, RUAN Rui. CMA blind equalization with variable momentum based on nonlinear transformation function [J]. Applied Mechanics and Materials, 2014, (602-605): 2658-2661.

[4] XIAO Ying, YIN Fu-liang. Blind equalization based on direction gradient algorithm under impulse noise environment [J]. WSEAS Transactions on Communications, 2014, (13):171-177.

[5] XIAO Ying, YIN Fuliang. Blind equalization based on RLS algorithm using adaptive forgetting factor for underwater acoustic channel [J]. China Ocean Engineering. 2014, 28(3): 401-408.

[6] HAN Ying-ge, GUO Ye-cai, LI Bao-kun et al. Momentum term and orthogonal wavelet-based blind equalization algorithm [J]. Journal of System Simulation, 2008, 20(6): 1559-1562.

[7] XU Jin-biao, WANG Yu-min. Selecting $\beta$ in "Stop and GO" decision-directed algorithm and its momentum algorithm [J]. Journal of Electronics, 1998, 20(3): 321-329.

[8] ZHANG Shu-xia, ZHANG Song-yang, JIANG Yu-zhong. Momentum constant modulus algorithm for blind channel equalization [J]. Journal of Naval University of Engineering, 2007, 19(2): 99-102.

[9] XING Li-kun, WU Long, GUO Ye-cai. Variable momentum factor odd symmetry error function blind equalization algorithm [J]. Computer Engineering and Application, 2010, 46(27): 117-119.

[10]A. M. Ragheb, M. Shoaib, S. Alshebeili, et al. Enhanced Blind Equalization for Optical DP-QAM in Finite Precision Hardware [J]. IEEE Photonics Technology Letters, 2015, 27(2): 181-184.

[11]Chung, Wonzoo1. Ill Convergence of Minimum Output Energy Infinite Impulse Response Equalizer for Digital Vestigial Sideband Signals [J]. International Journal of Adaptive Control and Signal Processing, 2012, 26(1): 73-83. 
[12] XIAO Ying, Li Min. Recursive Least Squares Fractionally-spaced blind equalization Algorithm for underwater acoustic communication [J]. Journal of Information and Computational Science, 2013, 10(18):6077-6084. 